\title{
Hosoya and Harary Polynomials of Hourglass and Rhombic Benzenoid Systems
}

\author{
Zhong-Lin Cheng, ${ }^{1}$ Ashaq Ali, ${ }^{2}$ Haseeb Ahmad $\left(D,{ }^{3,4}\right.$ Asim Naseem, \\ and Maqbool Ahmad Chaudhary ${ }^{2}$ \\ ${ }^{1}$ Teaching Department of Public Basic Course, Anhui International Studies University, Hefei 231201, China \\ ${ }^{2}$ Department of Mathematics and Statistics, University of Lahore, Lahore 54000, Pakistan \\ ${ }^{3}$ Department of Mathematics, Lahore Leads University, Lahore, Pakistan \\ ${ }^{4}$ Department of Mathematics, COMSATS University Islamabad, Lahore Campus, Lahore, Pakistan \\ ${ }^{5}$ Department of Mathematics, Government College University, Lahore, Pakistan \\ Correspondence should be addressed to Haseeb Ahmad; haseeb.ahmad@leads.edu.pk
}

Received 25 September 2019; Accepted 5 December 2019; Published 9 April 2020

Guest Editor: Shaohui Wang

Copyright @ 2020 Zhong-Lin Cheng et al. This is an open access article distributed under the Creative Commons Attribution License, which permits unrestricted use, distribution, and reproduction in any medium, provided the original work is properly cited.

\begin{abstract}
In the fields of chemical graph theory, topological index is a type of a molecular descriptor that is calculated based on the graph of a chemical compound. In 1947, Harry Wiener introduced "path number" which is now known as Wiener index and is the oldest topological index related to molecular branching. Hosoya polynomial plays a vital role in determining Wiener index. In this report, we compute the Hosoya polynomials for hourglass and rhombic benzenoid systems and recover Wiener and hyper-Wiener indices from them.
\end{abstract}

\section{Introduction}

Cheminformatics is a new branch of science which relates chemistry, mathematics, and computer sciences. Quantitative structure-activity (QSAR) and structure-property relationships (QSPR) are the main components of cheminformatics which are helpful to study physicochemical properties of chemical compounds [1-3].

A topological index is a numeric quantity associated with a graph which characterizes the topology of graph and is invariant under graph automorphism [4-8]. There are numerous applications of graph theory in the field of structural chemistry. The first well-known use of a topological index in chemistry was by Wiener in the study of paraffin boiling points [9]. After that, in order to explain physicochemical properties, various topological indices have been introduced.

The Hosoya polynomial of a graph is a generating function about distance distribution, introduced by Haruo Hosoya in 1988 [10]. This polynomial has many chemical applications [11]; in particular, Wiener index can be directly obtained from the polynomial and studied extensively [12-14].

The Wiener index was first introduced by Harold Wiener in 1947 to study the boiling points of paraffin [9]. It plays an important role in the so-called inverse structure-property relationship problems [15]. For more details about this topological polynomial and index, see the paper series and the references therein [16-20]. In this report, we study Hosoya polynomials, Wiener index, and hyper-Wiener index of hourglass and rhombic benzenoid systems.

\section{Preliminaries}

Definition 1 (simple graph). A simple graph $G=(V, E)$ is a finite nonempty set $V(G)$ of objects called vertices together with a (possibly empty) set $E(G)$ of unordered pairs of distinct vertices of $G$ called edges. 
Definition 2 (Hosoya polynomial [10]). The Hosoya polynomial of a connected graph $G$ is denoted by $H(G, x)$ and defined as follows:

$$
H(G, x)=\frac{1}{2} \sum_{v \in V(G)} \sum_{u \in V(G)} x^{d(v, u)},
$$

where $d(u, v)$ denotes the distance between vertices $u$ and $v$.

Definition 3 (Wiener index [9]). The Wiener index of a connected graph $G$ is denoted by $W(G)$ and defined as the sum of distances between all pairs of vertices in $G$, i.e., it can be formulated as follows:

$$
W(G)=\frac{1}{2} \sum_{v \in V(G)} \sum_{u \in V(G)} d(u, v) .
$$

Note that the first derivative of the Hosoya polynomial at $x=1$ is equal to the Wiener index:

$$
W(G)=\left.\frac{\partial H(G)}{\partial x}\right|_{x=1} .
$$

Definition 4 (modified Wiener index). The modified Wiener index of a connected graph $G$ is denoted by $W_{\lambda}(G)$ and defined as the sum of $\lambda$ power distances between all pairs of vertices in $G$, where $\lambda=1,2,3,4, \ldots$ i.e., it can be formulated as follows:

$$
W(G)=\frac{1}{2} \sum_{v \in V(G)} \sum_{u \in V(G)} d(u, v)^{\lambda}
$$

For detailed survey about this index, see [21-23].

Definition 5 (hyper-Wiener index). Hyper-Wiener index is another distance-based graph invariants used for predicting physicochemical properties of organic compounds [24]. The hyper-Wiener index was introduced by Randić [25] as follows:

$$
W W(G)=\frac{1}{2} \sum_{v \in V(G)} \sum_{u \in V(G)}\left(d(u, v)+d(u, v)^{2}\right) .
$$

Definition 6 (modified hyper-Wiener index). Modified hyper-Wiener index (see $[26,27])$ of a connected graph $G$ is denoted by $W W_{\lambda}(G)$ and defined as follows:

$$
W W_{\lambda}(G)=\frac{1}{2} \sum_{v \in V(G)} \sum_{u \in V(G)}\left(d(u, v)^{\lambda}+d(u, v)^{2 \lambda}\right),
$$

where $\lambda=1,2,3,4, \ldots$.

Definition 7 (Harary polynomial). The Harary polynomial (see $[28,29]$ ) of a connected graph $G$ is denoted by $h(G)$ and defined as follows:

$$
h(G)=\sum_{v \in V(G)} \sum_{u \in V(G)} \frac{1}{d(v, u)} x^{d(v, u)} .
$$

Definition 8 (generalized Harary index). The generalized Harary index (see $[30,31]$ ) of a connected graph $G$ is denoted by $h_{t}(G)$ and defined as follows:

$$
h_{t}(G)=\sum_{v \in V(G)} \sum_{u \in V(G)} \frac{1}{d(v, u)}+t,
$$

where $t=1,2,3,4, \ldots$.

Definition 9 (multiplicative Wiener index). The multiplicative wiener index (see $[32,33]$ ) of a connected graph $G$ is denoted by $\pi(G)$ and defined as follows:

$$
\pi(G)=\prod_{u, v \subseteq V(G)} d(u, v) .
$$

For detailed applications of topological indices in chemistry, we refer [34-41] and the references therein.

\section{Methodology}

To compute the Hosoya polynomial of a graph $G$, we need to compute number of pairs of vertices at distance $1,2,3, \ldots \operatorname{dia}(G), \quad$ where $\quad \operatorname{dia}(G)=\max \{d(u, v) ; u$, $v \in V(G)\}$. For this purpose, we use mathematical induction. Here, the $\operatorname{dia}\left(X_{n}\right)=4 n-1$ and $\operatorname{dia}\left(R_{n}\right)=2 n+1$. The general view of Hosoya polynomial is as below, where $d$ is the diameter of graph.

$$
H(G ; x)=a_{0}(n) x^{0}+a_{1}(n) x^{1}+a_{2}(n) x^{2}+\cdots+a_{d}(n) x^{d} .
$$

\section{Computational Results}

Benzenoid hydrocarbons play a vital role in our environment and in the food and chemical industries. Benzenoid molecular graphs are systems with deleted hydrogens. It is a connected geometric figure obtained by arranging congruent regular hexagons in a plane, so that two hexagons are either disjoint or have a common edge. This figure divides the plane into one infinite (external) region and a number of finite (internal) regions. All internal regions must be regular hexagons. Benzenoid systems are of considerable importance in theoretical chemistry because they are the natural graph representation of benzenoid hydrocarbons. A vertex of a hexagonal system belongs to, at most, three hexagons. A vertex shared by three hexagons is called an internal vertex.

Definition 10 (benzenoid hourglass system). Let $X_{n}$ denotes the benzenoid hourglass, which is obtained from two copies of a triangular benzenoid $T_{n}$ by overlapping their external hexagons (Figure 1).

4.1. Results for Benzenoid Hourglass System. The benzenoid hourglass system has $2\left(n^{2}+4 n-2\right)$ vertices and $3 n^{2}+9 n-$ 6 edges. 


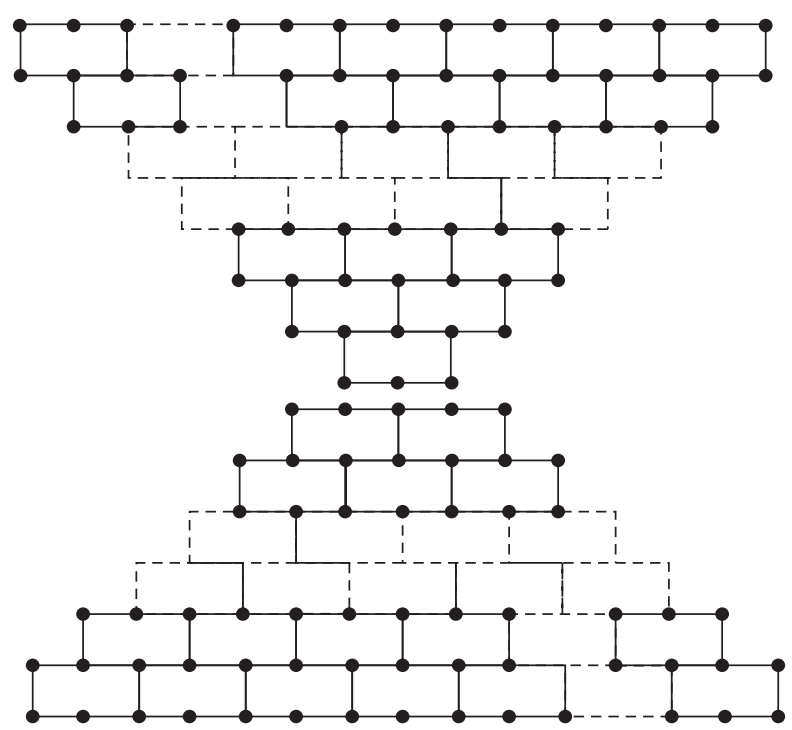

Figure 1: Benzenoid hourglass system $X_{n}$.

Theorem 1. For the benzenoid hourglass system $X_{n}$, we have

$$
\begin{aligned}
H\left(X_{n} ; x\right)= & 2\left(n^{2}+4 n-2\right)+\left(3 n^{2}+9 n-6\right)+\left(6 n^{2}+12 n-12\right) x^{2} \\
& +\sum_{m \equiv 1 \bmod (2), 1<m \leq 2 n}\left(\frac{19 m^{3}}{24}-3 m^{2} n-5 m^{2}+3 m n^{2}+12 m n+\frac{221 m}{24}-17\right) x^{m} \\
& +\sum_{m \equiv 0 \bmod (2), 2<m \leq 2 n}\left(\frac{19 m^{3}}{24}-3 m^{2} n-5 m^{2}+3 m n^{2}+12 m n+\frac{59 m}{6}-20\right) x^{m} \\
& +\sum_{m \equiv 0 \bmod (2), 2 \leq m \leq 2 n-2}\left(-\frac{m^{3}}{24}-\frac{m^{2} n}{4}+\frac{m n^{2}}{2}+\frac{n^{3}}{3}-m^{2}+4 n^{2}-\frac{35 m}{6}+\frac{23 n}{3}-4\right) x^{2 n+m} \\
& +\sum_{m \equiv 1 \bmod (2), 3 \leq m \leq 2 n-2}\left(\frac{m^{3}}{24}-\frac{m^{2} n}{4}-m^{2}+\frac{m n^{2}}{2}-\frac{143 m}{24}+\frac{n^{3}}{3}+4 n^{2}+\frac{101 n}{12}-5\right) x^{2 n+m} \\
& +\left(\frac{133 n}{6}+\frac{11(n-1)^{2}}{2}+\frac{(n-1)^{3}}{3}-\frac{127}{6}\right) x^{2 n+1}+\left(n^{2}+2\right) x^{4 n-1} \cdot
\end{aligned}
$$

Proof. To prove this theorem, we need to compute $\left|a_{m}(n)\right|$ where $m=1,2,3, \ldots, 4 n-1$. It is easy to verify that

$$
\begin{aligned}
& \left|a_{0}(n)\right|=|V|=2\left(n^{2}+4 n-2\right), \\
& \left|a_{1}(n)\right|=|E|=3 n^{2}+9 n-6, \\
& \left|a_{2}(n)\right|=6 n^{2}+12 n-12 .
\end{aligned}
$$

The remaining proof is divided into six parts which are according to the parity of $m$.

Case 1. $m \equiv 1 \bmod (2), 1<m \leq 2 n$.

It can be observed from Figure 1 that

$$
\begin{aligned}
& \left|a_{3}(1)\right|=0, \\
& \left|a_{3}(2)\right|=41, \\
& \left|a_{3}(3)\right|=95 \\
& \left|a_{3}(4)\right|=167 \\
& \left|a_{3}(5)\right|=257 .
\end{aligned}
$$

Now, one can conclude that

$$
\left|a_{3}(n)\right|=9(n-1)^{2}+27(n-1)+5 .
$$

Using a similar fashion, we have 


$$
\begin{aligned}
& \left|a_{5}(1)\right|=0, \\
& \left|a_{5}(2)\right|=0, \\
& \left|a_{5}(3)\right|=93, \\
& \left|a_{5}(4)\right|=183, \\
& \left|a_{5}(5)\right|=303, \\
& \left|a_{5}(6)\right|=453 .
\end{aligned}
$$

It implies that

$$
\left|a_{5}(n)\right|=15(n-2)^{2}+45(n-2)+33 .
$$

In a similar fashion, we infer

$$
\begin{aligned}
\left|a_{7}(n)\right| & =21(n-3)^{2}+63(n-3)+74, \\
\left|a_{9}(n)\right| & =27(n-4)^{2}+81(n-4)+130 \\
\left|a_{11}(n)\right| & =33(n-5)^{2}+99(n-5)+203 \\
\ldots &
\end{aligned}
$$

In terms of mathematical induction, we yield

$$
\begin{aligned}
\left|a_{m}(n)\right|= & 3 m\left(n-\frac{m}{2}+\frac{1}{2}\right)^{2}+9 m\left(n-\frac{m}{2}+\frac{1}{2}\right) \\
& +\frac{9((m / 2)-(1 / 2))^{2}}{2}+\frac{((m / 2)-(1 / 2))^{3}}{3} \\
& -\frac{217}{12}+\frac{73 m}{12}, \\
\left|a_{m}(n)\right|= & \frac{19 m^{2}}{24}-3 m^{2} n-5 m^{2}+3 m n^{2}+12 m n+\frac{221 m}{24}-17 .
\end{aligned}
$$

Case 2. $m \equiv 0 \bmod (2), 2<m \leq 2 n$.

It can be observed from Figure 1 that

$$
\begin{aligned}
& \left|a_{4}(1)\right|=0, \\
& \left|a_{4}(2)\right|=38, \\
& \left|a_{4}(3)\right|=98, \\
& \left|a_{4}(4)\right|=182, \\
& \left|a_{4}(5)\right|=290 .
\end{aligned}
$$

Now, one can conclude that

$$
\left|a_{4}(n)\right|=12(n-1)^{2}+24(n-1)+2 .
$$

By means of the same trick, we obtain

$$
\begin{aligned}
& \left|a_{6}(1)\right|=0, \\
& \left|a_{6}(2)\right|=0, \\
& \left|a_{6}(3)\right|=84, \\
& \left|a_{6}(4)\right|=174, \\
& \left|a_{6}(5)\right|=300, \\
& \left|a_{6}(6)\right|=462,
\end{aligned}
$$

which reveals that

$$
\left|a_{6}(n)\right|=18(n-2)^{2}+36(n-2)+30 .
$$

In light of the similar approach, we get

$$
\begin{aligned}
& \left|a_{8}(n)\right|=24(n-3)^{2}+48(n-3)+72, \\
& \left|a_{10}(n)\right|=30(n-4)^{2}+60(n-4)+130, \\
& \left|a_{12}(n)\right|=36(n-5)^{2}+72(n-5)+206,
\end{aligned}
$$

Hence, by mathematical induction, we have

$$
\begin{aligned}
\left|a_{m}(n)\right|= & 3 m\left(n-\frac{m}{2}+1\right)^{2}+6 m\left(n-\frac{m}{2}+1\right)^{2} \\
& +5\left(\frac{m}{2}-1\right)^{2}+\frac{((m / 2)-1)^{3}}{3}-\frac{74}{3}+\frac{16 m}{3}, \\
\left|a_{m}(n)\right|= & \frac{19 m^{3}}{24}-3 m^{2} n-5 m^{2}+3 m n^{2}+12 m n+\frac{59 m}{6}-20 .
\end{aligned}
$$

Case 3. $m \equiv 1 \bmod (2), 3 \leq m \leq 2 n-2$.

It can be observed from Figure 1 that

$$
\begin{aligned}
\left|a_{5}(1)\right| & =0, \\
\left|a_{7}(2)\right| & =0, \\
\left|a_{9}(3)\right| & =44, \\
\left|a_{11}(4)\right| & =41, \\
\left|a_{13}(5)\right| & =101, \\
\left|a_{15}(6)\right| & =177, \\
\left|a_{17}(7)\right| & =274 .
\end{aligned}
$$

Now, one can conclude that

$$
\left|a_{2 n+m}(n)\right|=\frac{(n-2)^{3}}{3}+\frac{15}{2}(n-2)^{2}+\frac{193}{6}(n-2)+4 .
$$

Using a similar fashion, we have

$$
\begin{aligned}
\left|a_{5}(1)\right| & =0, \\
\left|a_{7}(2)\right| & =0, \\
\left|a_{9}(3)\right| & =0, \\
\left|a_{11}(4)\right| & =0, \\
\left|a_{13}(5)\right| & =101, \\
\left|a_{15}(6)\right| & =150, \\
\left|a_{17}(7)\right| & =254, \\
\left|a_{19}(8)\right| & =383, \\
\left|a_{21}(9)\right| & =539 .
\end{aligned}
$$

It implies that 


$$
\left|a_{2 n+m}(n)\right|=\frac{(n-3)^{3}}{3}+\frac{19}{2}(n-3)^{2}+\frac{301}{6}(n-3)+9
$$

In a similar fashion, we infer

$\left|a_{2 n+m}(n)\right|=\frac{(n-4)^{3}}{3}+\frac{23}{2}(n-4)^{2}+\frac{433}{6}(n-4)+16$,

$\left|a_{2 n+m}(n)\right|=\frac{(n-5)^{3}}{3}+\frac{27}{2}(n-5)^{2}+\frac{589}{6}(n-5)+25$,

$\left|a_{2 n+m}(n)\right|=\frac{(n-6)^{3}}{3}+\frac{31}{2}(n-6)^{2}+\frac{769}{6}(n-6)+36$

In terms of mathematical induction, we yield

$$
\begin{aligned}
\left|a_{2 n+m}(n)\right|= & \left(\frac{(n-((m+1) / 2))^{3}}{3}+\frac{1}{2}\left(4\left(\frac{m-1}{2}\right)+11\right)\right. \\
& \cdot\left(n-\left(\frac{m+1}{2}\right)\right)^{2}+\left(\frac{1}{6}\right)\left(12\left(\frac{m-1}{2}\right)^{2}\right. \\
& \left.+72\left(\frac{m-1}{2}\right)+109\right)\left(n-\left(\frac{m+1}{2}\right)\right) \\
& \left.+\left(\frac{m-1}{2}\right)^{2}+2\left(\frac{m-1}{2}\right)+1\right), \\
& -\frac{m^{3}}{24}-\frac{m^{2} n}{4}-m^{2}+\frac{m n^{2}}{2}-\frac{143 m}{24}+\frac{n^{3}}{3} \\
& +4 n^{2}+\frac{101 n}{12}-5 .
\end{aligned}
$$

Case 4. $m \equiv 0 \bmod (2), 2 \leq m \leq 2 n-2$.

It can be observed from Figure 1 that

$$
\begin{aligned}
& \left|a_{4}(1)\right|=0, \\
& \left|a_{6}(2)\right|=16, \\
& \left|a_{8}(3)\right|=108, \\
& \left|a_{10}(4)\right|=41, \\
& \left|a_{12}(5)\right|=180, \\
& \left|a_{14}(6)\right|=272, \\
& \left|a_{16}(7)\right|=386 .
\end{aligned}
$$

Now, one can conclude that

$$
\left|a_{2 n+m}(n)\right|=\frac{(n-1)^{3}}{3}+6(n-1)^{2}+\frac{53}{3}(n-1)-8 .
$$

Using a similar fashion, we have

$$
\begin{aligned}
& \left|a_{4}(1)\right|=0, \\
& \left|a_{6}(2)\right|=0, \\
& \left|a_{8}(3)\right|=0 \\
& \left|a_{10}(4)\right|=28 \\
& \left|a_{12}(5)\right|=86, \\
& \left|a_{14}(6)\right|=164, \\
& \left|a_{16}(7)\right|=264
\end{aligned}
$$

It implies that

$\left|a_{2 n+m}(n)\right|=\frac{(n-2)^{3}}{3}+8(n-2)^{2}+\frac{95}{3}(n-2)-12$.

In a similar fashion, we infer

$$
\begin{aligned}
& \left|a_{2 n+m}(n)\right|=\frac{(n-3)^{3}}{3}+10(n-3)^{2}+\frac{149}{3}(n-4)-16, \\
& \left|a_{2 n+m}(n)\right|=\frac{(n-4)^{3}}{3}+12(n-4)^{2}+\frac{215}{3}(n-5)-20, \\
& \left|a_{2 n+m}(n)\right|=\frac{(n-5)^{3}}{3}+14(n-5)^{2}+\frac{293}{3}(n-6)-24, \\
& \ldots
\end{aligned}
$$

In terms of mathematical induction, we yield

$$
\begin{aligned}
\left|a_{2 n+m}(n)\right|= & \left(\frac{(n-(m / 2))^{3}}{3}+\left(2\left(\frac{m}{2}\right)+4\right)\left(n-\left(\frac{m}{2}\right)\right)^{2}\right. \\
& +\left(\frac{6(m / 2)^{2}+24(m / 2)+23}{3}\right)\left(n-\left(\frac{m}{2}\right)\right) \\
& -4\left(\frac{m}{2}\right)-4,
\end{aligned}
$$

$$
\begin{aligned}
\left|a_{2 n+m}(n)\right|= & (m+4)\left(\frac{m}{2}-n\right)^{2}-\left(\frac{(m / 2)-n}{2}\right)^{3} \\
& -2 m-\left(\frac{m}{2}-n\right)\left(\frac{m^{2}}{2}+4 m+\frac{23}{3}\right)-4, \\
\left|a_{2 n+m}(n)\right|= & -\frac{m^{3}}{24}-\frac{m^{2} n}{4}+\frac{m n^{2}}{2}+\frac{n^{3}}{3}-m^{2}+4 n^{2} \\
& -\frac{35 m}{6}+\frac{23 n}{3}-4 .
\end{aligned}
$$

Case 5. $m=2 n+1$.

It can be observed from Figure 1 that 


$$
\begin{aligned}
& \left|a_{5}(1)\right|=0, \\
& \left|a_{7}(2)\right|=29, \\
& \left|a_{9}(3)\right|=70, \\
& \left|a_{11}(4)\right|=126, \\
& \left|a_{13}(5)\right|=199, \\
& \left|a_{15}(6)\right|=291, \\
& \left|a_{17}(7)\right|=404 .
\end{aligned}
$$

Now, one can conclude that

$$
\begin{aligned}
& \left|a_{2 n+1}(n)\right|=\frac{(n-1)^{3}}{3}+\frac{11}{2}(n-1)^{2}+\frac{133}{6}(n-1)+1, \\
& \left|a_{2 n+1}(n)\right|=\frac{133 n}{6}+\frac{11(n-1)^{2}}{2}+\frac{(n-1)^{3}}{3}-\frac{127}{6} .
\end{aligned}
$$

Case 6. $m=4 n-1$.
It can be observed from Figure 1 that

$$
\begin{aligned}
& \left|a_{3}(1)\right|=3, \\
& \left|a_{7}(2)\right|=6, \\
& \left|a_{11}(3)\right|=11, \\
& \left|a_{15}(4)\right|=18, \\
& \left|a_{19}(5)\right|=27, \\
& \left|a_{23}(6)\right|=38, \\
& \left|a_{27}(7)\right|=51 .
\end{aligned}
$$

Now, one can conclude that

$$
\left|a_{4 n-1}(n)\right|=n^{2}+2 .
$$
result.

By what have been mentioned above, we get our required

Theorem 2. For the benzenoid hourglass system $X_{n}$, the Harary polynomial is given by

$$
\begin{aligned}
h\left(X_{n} ; x\right)= & \left(3 n^{2}+9 n-6\right) x+\left(3 n^{2}+6 n-6\right) x^{2} \\
& +\sum_{m \equiv 1 \bmod (2), 1<m \leq 2 n} \frac{1}{m}\left(\frac{19 m^{3}}{24}-3 m^{2} n-5 m^{2}+3 m n^{2}+12 m n+\frac{221 m}{24}-17\right) x^{m} \\
& +\sum_{m \equiv 0 \bmod (2), 2<m \leq 2 n} \frac{1}{m}\left(\frac{19 m^{3}}{24}-3 m^{2} n-5 m^{2}+3 m n^{2}+12 m n+\frac{59 m}{6}-20\right) x^{m} \\
& +\sum_{m \equiv 0 \bmod (2), 2 \leq m \leq 2 n-2} \frac{1}{2 n+m}\left((m+4)\left(\frac{m}{2}-n\right)^{2}-\frac{((m / 2)-n)^{3}}{3}-2 m-\left(\frac{m}{2}-n\right)\left(\frac{m^{2}}{2}+4 m+\frac{23}{3}\right)-4\right) x^{2 n+m} \\
& +\sum_{m \equiv 1 \bmod (2), 3 \leq m \leq 2 n-2} \frac{1}{2 n+m}\left(-\frac{m^{3}}{24}-\frac{m^{2} n}{4}-m^{2}+\frac{m n^{2}}{2}-\frac{143 m}{24}+\frac{n^{3}}{3}+4 n^{2}+\frac{101 n}{12}-5\right) x^{2 n+m} \\
& +\frac{1}{2 n+1}\left(\frac{133 n}{6}+\frac{11(n-1)^{2}}{2}+\frac{(n-1)^{3}}{3}-\frac{127}{6}\right) x^{2 n+1}+\frac{1}{4 n-1}\left(n^{2}+2\right) x^{4 n-1} .
\end{aligned}
$$

Proof. From the information about the number of pairs of vertices at different distances given in Theorem 1 , it is easy to get our desired result.

Theorem 3. For the benzenoid hourglass system $X_{n}$, we have

(i) $W_{\lambda}\left(X_{n}\right)=\left(3 n^{2}+9 n-6\right)+\left(6 n^{2}+12 n-12\right) 2^{\lambda}+$ $\sum_{m=1 \bmod (2), 1<m \leq 2 n}\left(\left(19 m^{3} / 24\right)-3 m^{2} n-5 m^{2}+3 m n^{2}\right.$ $+12 m n+(221 m / 24)-17) m^{\lambda}+$ $\sum_{m=0 \bmod (2), 2<m \leq 2 n}\left(\left(19 m^{3} / 24\right)-3 m^{2} n-5 m^{2}+\right.$ $\left.3 m n^{2}+12 m n+(59 m / 6)-20\right) m^{\lambda}+$ $\sum_{m=0 \bmod (2), 2 \leq m \leq 2 n-2}\left((m+4)((m / 2)-n)^{2}-\right.$ $\left(((m / 2)-n)^{3} / 3\right)-2 m-((m / 2)-n)\left(\left(m^{2} / 2\right)+\right.$ $\left.4 m+(23 / 3))-4(2 n+m)^{\lambda}\right)+$ $+\sum_{m=1 \bmod (2), 3 \leq m \leq 2 n-2}\left(-\left(m^{3} / 24\right)-\left(m^{2} n / 4\right)-m^{2}+\right.$ $\left(m n^{2} / 2\right)-(143 m / 24)+\left(n^{3} / 3\right)+4 n^{2}+(101 n / 12)-$ 5) $(2 n+m)^{\lambda}+((133 n / 6)+$

$\left(11(n-1)^{2} / 2\right)+\left((n-1)^{3} / 3\right)-$ $(127 / 6))(2 n+1)^{\lambda}+\left(n^{2}+2\right)(4 n-1)^{\lambda}$.

(ii) $W W_{\lambda}\left(X_{n}\right)=\left(3 n^{2}+9 n-6\right)\left(1^{\lambda}+1^{2 \lambda}\right)+$ $\left(6 n^{2}+12 n-12\right)\left(2^{\lambda}+2^{2 \lambda}\right)+$ $\sum_{m=1 \bmod (2), 1<m \leq 2 n}\left(\left(19 m^{3} / 24\right)-3 m^{2} n-5 m^{2}+\right.$ $\left.3 m n^{2}+12 m n+(221 m / 24)-17\right)\left(m^{\lambda}+m^{2 \lambda}\right)+$ 
$\sum_{m=0 \bmod (2), 2<m \leq 2 n}\left(\left(\left(19 m^{3} / 24\right)-3 m^{2} n-5 m^{2}+\right.\right.$ $\left.\left.3 m n^{2}+12 m n+(59 m / 6)-20\right)\left(m^{\lambda}+m^{2 \lambda}\right)\right)+$ $\sum_{m \equiv 0 \bmod (2), 2 \leq m \leq 2 n-2}\left((m+4)((m / 2)-n)^{2}-\right.$ $\left(((m / 2)-n)^{3} / 3\right)-2 m-((m / 2)-n)\left(\left(m^{2} / 2\right)+\right.$ $4 m+(23 / 3))-4)\left((2 n+m)^{\lambda}+(2 n+m)^{2 \lambda}\right)+$ $\sum_{m \equiv 1 \bmod (2), 3 \leq m \leq 2 n-2}\left(-\left(m^{3} / 24\right)-\left(m^{2} n / 4\right)-m^{2}+\right.$ $\left(m n^{2} / 2\right)-(143 m / 24)+\left(n^{3} / 3\right)+4 n^{2}+(101 n / 12)-$ 5) $\left((2 n+m)^{\lambda}+(2 n+m)^{2 \lambda}\right)+((133 n / 6)+$ $\left(11(n-1)^{2} / 2+\left((n-1)^{3} / 3\right)\right)-$

$(127 / 6))\left((2 n+1)^{\lambda}+(2 n+1)^{2 \lambda}\right)+\left(n^{2}+\right.$ 2) $\left((4 n-1)^{\lambda}+(4 n-1)^{2 \lambda}\right)$.

(iii) $H_{t}\left(X_{n}\right)=\left(3 n^{2}+9 n-6\right)(1 /(1+t))+$ $\left(6 n^{2}+12 n-12\right)(1 /(2+t))+$ $\sum_{m \equiv 1 \bmod (2), 1<m \leq 2 n}\left(\left(19 m^{3} / 24\right)-3 m^{2} n-5 m^{2}+\right.$ $\left.3 m n^{2}+12 m n+(221 m / 24)-17\right)(1 / m+t)+$ $\sum_{m \equiv 0 \bmod (2), 2<m \leq 2 n}\left(\left(19 m^{3} / 24\right)-3 m^{2} n-5 m^{2}+\right.$ $\left.3 m n^{2}+12 m n+(59 m / 6)-20\right)(1 / m+t)+$ $\sum_{m \equiv 0 \bmod (2), 2 \leq m \leq 2 n-2}\left((m+4)((m / 2)-n)^{2}-\right.$ $\left.((m / 2)-n)^{3} / 3\right)-2 m((m / 2)-n)((m / 2)-$ n) $\left(\left(m^{2} / 2\right) 4 m+(23 / 3)-4\right)(1 / 2 n+m+t)+$ $\sum_{m \equiv 1 \bmod (2), 3 \leq m \leq 2 n-2}\left(-\left(m^{3} / 24\right)-\left(m^{2} n / 4\right)-m^{2}+\right.$ $\left(m n^{2} / 2\right)-(143 m / 24)+\left(n^{3} / 3\right)+4 n^{2}+(101 n / 12)-$ 5) $(1 / 2 n+m+t)+\left((133 n / 6)+\left(11(n-1)^{2} / 2\right)+\right.$ $\left.\left((n-1)^{3} / 3\right)-(127 / 6)\right)(1 / 2 n+1+t)+\left(n^{2}+\right.$ 2) $(1 / 4 n-1+t)$.

(iv) $\pi\left(X_{n}\right)=1^{\left(3 n^{2}+9 n-6\right)} \times 2^{\left(6 n^{2}+12 n-12\right)} \times$

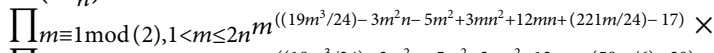
$\prod_{m \equiv 0 \bmod (2), 2<m \leq 2 n^{m}} m^{\left(\left(19 m^{3} / 24\right)-3 m^{2} n-5 m^{2}+3 m n^{2}+12 m n+(59 m / 6)-20\right)} \times$ $\prod_{m \equiv 0 \bmod (2), 2 \leq m \leq 2 n-2}(2 n+m)^{\left((m+4)((m / 2)-n)^{2}-\left(((m / 2)-n)^{3} /\right.\right.}$ $3)-2 m-((m / 2)-n)\left(\left(m^{2} / 2\right)+4 m+(23 / 3)\right)-$ 4) $\times \prod_{m \equiv 1 \bmod (2), 3 \leq m \leq 2 n-2}(2 n+m)^{\left(-\left(m^{3} / 24\right)-\left(m^{2} n / 4\right)\right.}$ $-m^{2}+\left(m n^{2} / 2\right)-(143 m / 24)+\left(n^{3} / 3\right)+4 n^{2}+$ $(101 n / 12)-5) \times(2 n+1)^{\left((133 n / 6)+\left(11(n-1)^{2} / 2\right)+\right.}$ $\left.\left((n-1)^{3} / 3\right)-(127 / 6)\right) \times(4 n-1)^{\left(n^{2}+2\right)}$.

From the above theorem, we get the following results immediately.

Corollary 1. For the benzenoid hourglass system $X_{n}$, we have

$$
W\left(X_{n}\right)=\frac{52 n^{5}}{15}+\frac{80 n^{4}}{3}+34 n^{3}-\frac{116 n^{2}}{3}-\frac{37 n}{15}+4
$$

Corollary 2. For the benzenoid hourglass system $X_{n}$, we have

$$
\begin{aligned}
W W\left(X_{n}\right)= & \frac{4 n^{5}}{3}+36 n^{4}+\frac{217 n^{3}}{3}+92 n^{2}+\frac{31 n}{3}-116 \\
& +\sum_{m \equiv 1 \bmod (2), 1<m \leq 2 n} m(m+1)\left(\frac{19 m^{3}}{24}-3 m^{2} n-5 m^{2}+3 m n^{2}+12 m n+\frac{221 m}{24}-17\right) \\
& +\sum_{m \equiv 0 \bmod (2), 2<m \leq 2 n} m(m+1)\left(\frac{19 m^{3}}{24}-3 m^{2} n-5 m^{2}+3 m n^{2}+12 m n+\frac{59 m}{6}-20\right) \\
& +\sum_{m \equiv 0 \bmod (2), 2 \leq m \leq 2 n-2}\left(2 n+m+(2 n+m)^{2}\right)\left((m+4)\left(\frac{m}{2}-n\right)^{2}-\frac{((m / 2)-n)^{3}}{3}-2 m-\left(\frac{m}{2}-n\right)\left(\frac{m^{2}}{2}+4 m+\frac{23}{3}\right)-4\right) \\
& +\sum_{m \equiv 1 \bmod (2), 3 \leq m \leq 2 n-2}\left(2 n+m+(2 n+m)^{2}\right)\left(-\frac{m^{3}}{24}-\frac{m^{2} n}{4}-m^{2}+\frac{m n^{2}}{2}-\frac{143 m}{24}+\frac{n^{3}}{3}+4 n^{2}+\frac{101 n}{12}-5\right) .
\end{aligned}
$$

Corollary 3. The Harary index of benzenoid hourglass graph

$X_{n}$ is as follows: 


$$
\begin{aligned}
h\left(X_{n}\right)= & \frac{296 n^{4}+910 n^{3}-161 n^{2}-667 n+180}{6\left(8 n^{2}+2 n-1\right)} \\
& +\sum_{m \equiv 1 \bmod (2), 1<m \leq 2 n} \frac{1}{m}\left(\frac{19 m^{3}}{24}-3 m^{2} n-5 m^{2}+3 m n^{2}+12 m n+\frac{221 m}{24}-17\right) \\
& +\sum_{m \equiv 0 \bmod (2), 2<m \leq 2 n} \frac{1}{m}\left(\frac{19 m^{3}}{24}-3 m^{2} n-5 m^{2}+3 m n^{2}+12 m n+\frac{59 m}{6}-20\right) \\
& +\sum_{m \equiv 0 \bmod (2), 2 \leq m \leq 2 n-2} \frac{1}{2 n+m}\left((m+4)\left(\frac{m}{2}-n\right)^{2}-\frac{((m / 2)-n)^{3}}{3}-2 m-\left(\frac{m}{2}-n\right)\left(\frac{m^{2}}{2}+4 m+\frac{23}{3}\right)-4\right) \\
& +\sum_{m \equiv 1 \bmod (2), 3 \leq m \leq 2 n-2} \frac{1}{2 n+m}\left(-\frac{m^{3}}{24}-\frac{m^{2} n}{4}-m^{2}+\frac{m n^{2}}{2}-\frac{143 m}{24}+\frac{n^{3}}{3}+4 n^{2}+\frac{101 n}{12}-5\right) .
\end{aligned}
$$

4.2. Benzenoid Rhombus System. Consider a benzenoid system in which hexagons are arranged to form a rhombic shape, say, $R_{n}$, where $n$ represents number of hexagons along each boundary of the rhombic as given in Figure 2 .
Lemma 1. The benzenoid rhombus system has $2 n^{2}+4 n$ vertices and $3 n^{2}+4 n-1$ edges.

Theorem 4. For the benzenoid rhombus system $R_{n}$, we have

$$
\begin{aligned}
H\left(R_{n} ; x\right)= & 2 n^{2}+4 n+\left(3 n^{2}+4 n-1\right) x+\left(6 n^{2}+4 n-4\right) x^{2} \\
& +\sum_{m \equiv 1 \bmod (2), 1<m \leq 2 n+1}\left(3 m n^{2}-2 m^{2} n+\frac{7 m^{3}}{24}+6 m n-2 m^{2}+\frac{41 m}{24}-1\right) x^{m} \\
& +\sum_{m \equiv 0 \bmod (2), 2<m \leq 2 n}\left(3 m n^{2}-2 m^{n}+\frac{7 m^{3}}{24}+6 m n-2 m^{2}+\frac{11 m}{6}-2\right) x^{m} \\
& +\sum_{m \equiv 1 \bmod (2), 1 \leq m \leq 2 n-3}\left(\frac{(-m+2 n-2)^{3}}{24}+\frac{(-m+2 n-2)^{2}}{2}+\frac{23(-m+2 n-2)}{24}-\frac{1}{2}\right) x^{2 n+m+2} \\
& +\sum_{m \equiv 0 \bmod (2), 2 \leq m \leq 2 n-2} \frac{(-m+2 n)^{3}}{24}\left(+\frac{(-m+2 n)^{2}}{2}+\frac{5(-m+2 n)}{6}\right) x^{2 n+m} .
\end{aligned}
$$

Proof. To prove this theorem, we need to compute $\left|a_{m}(n)\right|$ where $p=1,2,3, \ldots, 2 n+1$. It is easy to verify that

$$
\begin{aligned}
& \left|a_{0}(n)\right|=|V|=2 n^{2}+4 n, \\
& \left|a_{1}(n)\right|=|E|=3 n^{2}+4 n-1, \\
& \left|a_{2}(n)\right|=6 n^{2}+4 n-4 .
\end{aligned}
$$

The remaining proof is divided into two parts which are according to the parity of $m$.

Case 7. $m \equiv 1 \bmod (2), 1<m \leq 2 n+1$.

It can be observed from Figure 2 that

$$
\begin{aligned}
& \left|a_{3}(1)\right|=3, \\
& \left|a_{3}(2)\right|=30, \\
& \left|a_{3}(3)\right|=75, \\
& \left|a_{3}(4)\right|=138, \\
& \left|a_{3}(5)\right|=219 .
\end{aligned}
$$

Now, one can conclude that

$$
\left|a_{3}(n)\right|=9 n^{2}-6 \text {. }
$$

In a similar fashion, we have 


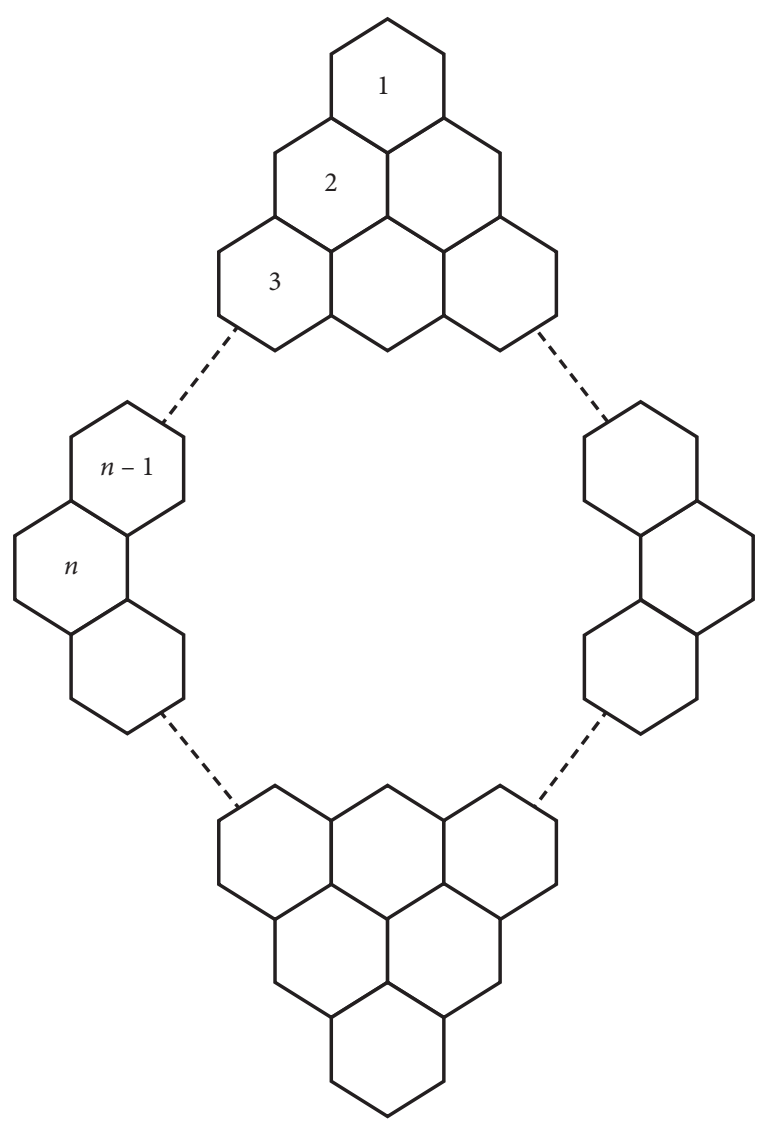

Figure 2: Benzenoid rhombus system $R_{n}$.

$$
\begin{aligned}
& \left|a_{5}(1)\right|=0, \\
& \left|a_{5}(2)\right|=24, \\
& \left|a_{5}(3)\right|=76, \\
& \left|a_{5}(4)\right|=152, \\
& \left|a_{5}(5)\right|=252, \\
& \left|a_{5}(6)\right|=376 .
\end{aligned}
$$

It implies that

$$
\left|a_{5}(n)\right|=15(n-1)^{2}+10(n-1)-11
$$

In a similar fashion, we infer

$$
\begin{gathered}
\left|a_{7}(n)\right|=21(n-2)^{2}+28(n-2)-15, \\
\left|a_{9}(n)\right|=27(n-3)^{2}+54(n-3)-16, \\
\left|a_{11}(n)\right|=33(n-4)^{2}+88(n-4)-12,
\end{gathered}
$$

In terms of mathematical induction, we yield

$$
\begin{aligned}
\left|a_{m}(n)\right|= & 3 m\left(n-\left(\frac{m-3}{2}\right)\right)^{2} \\
& +\left(m^{2}-3 m\right)\left(n-\left(\frac{m-3}{2}\right)\right) \\
& +\frac{1}{24} m^{3}-\frac{1}{2} m^{2}-\frac{13}{24} m-1, \\
\left|a_{m}(n)\right|= & 3 m n^{2}-2 m^{2} n+\frac{7 m^{3}}{24}+6 m n-2 m^{2}+\frac{41 m}{24}-1 .
\end{aligned}
$$

Case 8. $m \equiv 0 \bmod (2), 2<m \leq 2 n$.

It can be observed from Figure 2 that

$$
\begin{aligned}
& \left|a_{4}(1)\right|=0, \\
& \left|a_{4}(2)\right|=24, \\
& \left|a_{4}(3)\right|=76, \\
& \left|a_{4}(4)\right|=152, \\
& \left|a_{4}(5)\right|=252 .
\end{aligned}
$$

Now, one can conclude that

$$
\left|a_{4}(n)\right|=12(n-1)^{2}+16(n-1)-4 \text {. }
$$

By means of the same trick, we obtain

$$
\begin{aligned}
& \left|a_{6}(1)\right|=0, \\
& \left|a_{6}(2)\right|=0, \\
& \left|a_{6}(3)\right|=54, \\
& \left|a_{6}(4)\right|=144, \\
& \left|a_{6}(5)\right|=270, \\
& \left|a_{6}(5)\right|=432,
\end{aligned}
$$

which reveals that

$$
\left|a_{6}(n)\right|=18(n-2)^{2}+36 *(n-2) .
$$

In light of the similar approach, we get

$$
\begin{aligned}
& \left|a_{8}(n)\right|=24(n-3)^{2}+64(n-3)+10, \\
& \left|a_{10}(n)\right|=30(n-4)^{2}+100(n-4)+28, \\
& \left|a_{12}(n)\right|=36(n-5)^{2}+144(n-5)+56
\end{aligned}
$$

Hence, by mathematical induction, we have

$$
\begin{aligned}
\left|a_{m}(n)\right|= & 3 m\left(n-\frac{m}{2}+1\right)^{2}+m^{2}\left(n-\frac{m}{2}+1\right) \\
& +\frac{1}{24} m^{3}-\frac{7}{6} m-2, \\
\left|a_{m}(n)\right|= & 3 m n^{2}-2 m^{n}+\frac{7 m^{3}}{24}+6 m n-2 m^{2}+\frac{11 m}{6}-2 .
\end{aligned}
$$


Now for $m=2 n+2$ to $m=4 n-1$, we will generalize in this way. By observing Table 1, values in italics show the distances from $2 n+2$ to $4 n-1$, but the values in the table are in descending order, so first we generalized this in ascending order and then reverse its order as required, let $p_{i}$ be the values in ascending order as follows (from Table 1).

$$
\begin{aligned}
& p_{1}=1, \\
& p_{2}=4, \\
& p_{3}=8, \\
& p_{4}=14, \\
& p_{5}=22, \\
& p_{6}=32, \\
& p_{7}=45, \\
& p_{8}=60, \\
& p_{9}=79, \\
& p_{10}=100 .
\end{aligned}
$$

Case 9. $i \equiv 1 \bmod (2)$.

Hence, one can conclude that

$$
p_{i}=\frac{i^{3}}{24}+\frac{i^{2}}{2}+\frac{23 i}{24}-\frac{1}{2} \text {. }
$$

For $m \equiv 1 \bmod (2), 1 \leq m \leq 2 n-3$.

So to reverse its order put $i=(-m+2 n-2)$, we get

$$
\begin{aligned}
\left|a_{m}(n)\right|= & \frac{(-m+2 n-2)^{3}}{24}+\frac{(-m+2 n-2)^{2}}{2} \\
& +\frac{23(-m+2 n-2)}{24}-\frac{1}{2} .
\end{aligned}
$$

Case 10. $i \equiv 0 \bmod (2)$.

Hence, one can conclude that

$$
p_{i}=\frac{i^{3}}{24}+\frac{i^{2}}{2}+\frac{5 i}{6} \text {. }
$$

For $m \equiv 0 \bmod (2), 2 \leq m \leq 2 n-2$.

So to reverse its order put $i=(-m+2 n)$, we get

$$
\left|a_{m}(n)\right|=\frac{(-m+2 n)^{3}}{24}+\frac{(-m+2 n)^{2}}{2}+\frac{5(-m+2 n)}{6} \text {. }
$$
result.

By what has been mentioned above, we get our desired

Theorem 5. For the benzenoid rhombus system $R_{n}$, the Harary polynomial is as follows:

$$
\begin{aligned}
h\left(R_{n} ; x\right)= & \left(3 n^{2}+4 n-1\right) x+\left(3 n^{2}+2 n-2\right) x^{2} \\
& +\sum_{m \equiv 1 \bmod (2), 1<m \leq 2 n+1} \frac{1}{m}\left(3 m n^{2}-2 m^{2} n+\frac{7 m^{3}}{24}+6 m n-2 m^{2}+\frac{41 m}{24}-1\right) x^{m} \\
& +\sum_{m \equiv 0 \bmod (2), 2<m \leq 2 n} \frac{1}{m}\left(3 m n^{2}-2 m^{n}+\frac{7 m^{3}}{24}+6 m n-2 m^{2}+\frac{11 m}{6}-2\right) x^{m} \\
& +\sum_{m \equiv 1 \bmod (2), 1 \leq m \leq 2 n-3} \frac{1}{2 n+m+2}\left(\frac{(-m+2 n-2)^{3}}{24}+\frac{(-m+2 n-2)^{2}}{2}+\frac{23(-m+2 n-2)}{24}-\frac{1}{2}\right) x^{2 n+m+2} \\
& +\sum_{m \equiv 0 \bmod (2), 2 \leq m \leq 2 n-2} \frac{1}{2 n+m}\left(\frac{(-m+2 n)^{3}}{24}+\frac{(-m+2 n)^{2}}{2}+\frac{5(-m+2 n)}{6}\right) x^{2 n+m} .
\end{aligned}
$$

Proof. From the information about the number of pairs of vertices at different distances given in Theorem 4, one can easily get this result.

Theorem 6. For the benzenoid rhombus system $R_{n}$, we have

(i)

$$
\begin{aligned}
& W_{\lambda}\left(R_{n}\right)=\left(3 n^{2}+4 n-1\right)+\left(6 n^{2}+4 n-4\right) 2^{\lambda} \\
& \sum_{m \equiv 1 \bmod (2), 1<m \leq 2 n+1}\left(3 m n^{2}-2 m^{2} n+\left(7 m^{3} / 24\right)+\right. \\
& \left.6 m n-2 m^{2}+(41 m / 24)-1\right) m^{\lambda}+ \\
& \sum_{m \equiv 0 \bmod (2), 2<m \leq 2 n}\left(3 m n^{2}-2 m^{n}+\left(7 m^{3} / 24\right)+6 m n-\right.
\end{aligned}
$$

$$
\begin{aligned}
& \left.2 m^{2}+11 m / 6-2\right) m^{\lambda}+\sum_{m \equiv 1 \bmod (2), 1 \leq m \leq 2 n-3} \\
& \left(\left((-m+2 n-2)^{3} / 24\right)+\left((-m+2 n-2)^{2} / 2\right)+\right. \\
& (23(-m+2 n-2) / 24)-(1 / 2))(2 n+m+2)^{\lambda}+ \\
& \sum_{m=0 \bmod (2), 2 \leq m \leq 2 n-2}\left(\left((-m+2 n)^{3} / 24\right)+((-m+\right. \\
& \left.\left.2 n)^{2} / 2\right)+(5(-m+2 n) / 6)\right)(2 n+m)^{\lambda} .
\end{aligned}
$$

(ii) $W W_{\lambda}\left(R_{n}\right)=\left(3 n^{2}+4 n-1\right)\left(1^{\lambda}+1^{2 \lambda}\right)+\left(6 n^{2}+4 n-4\right)$ $\left(2^{\lambda}+2^{2 \lambda}\right)+\sum_{m \equiv 1 \bmod (2), 1<m \leq 2 n+1}\left(3 m n^{2}-2 m^{2} n+\right.$ $\left.\left(7 m^{3} / 24\right)+6 m n-2 m^{2}+(41 m / 24)-1\right)\left(m^{\lambda}+m^{2 \lambda}\right)+$ $\sum_{m \equiv 0 \bmod (2), 2<m \leq 2 n}\left(3 m n^{2}-2 m^{n}+\left(7 m^{3} / 24\right)+6 m n-\right.$ 
TABle 1: Number of pair of vertices at different distances.

\begin{tabular}{lcccccc}
\hline Distance $(m)$ & & \multicolumn{5}{c}{$n$} \\
& 2 & 3 & 4 & 5 & 6 & 7 \\
\hline 1 & 19 & 38 & 63 & 94 & 131 & 174 \\
2 & 28 & 62 & 108 & 166 & 236 & 318 \\
3 & 30 & 75 & 138 & 219 & 318 & 435 \\
4 & 24 & 76 & 152 & 252 & 376 & 524 \\
5 & 14 & 69 & 154 & 269 & 414 & 589 \\
6 & 4 & 54 & 144 & 270 & 432 & 630 \\
7 & 1 & 34 & 125 & 258 & 433 & 650 \\
8 & & 14 & 98 & 234 & 418 & 650 \\
9 & & 8 & 65 & 200 & 389 & 632 \\
10 & & 4 & 32 & 158 & 348 & 598 \\
11 & & 1 & 22 & 109 & 296 & 549 \\
12 & & & 14 & 60 & 236 & 488 \\
13 & & & 8 & 45 & 168 & 415 \\
14 & & & 4 & 32 & 100 & 334 \\
15 & & & 1 & 22 & 79 & 244 \\
16 & & & & 14 & 60 & 154 \\
17 & & & & 8 & 45 & 126 \\
18 & & & & 4 & 32 & 100 \\
19 & & & & 1 & 22 & 79 \\
20 & & & & & 14 & 60 \\
21 & & & & & & 45 \\
22 & & & & & & 32 \\
23 & & & & & & 22 \\
24 & & & & & & 14 \\
25 & & & & & & \\
27 & & & & & & \\
\hline
\end{tabular}

$\left.2 m^{2}+(11 m / 6)-2\right)\left(m^{\lambda}+m^{2 \lambda}\right)+\quad \sum_{m \equiv 1 \bmod (2)}$, $1 \leq m \leq 2 n-3\left(\left((-m+2 n-2)^{3} / 24\right)+((-m+2 n-\right.$ $\left.2)^{2} / 2\right)+(23(-m+2 n-2) / 24)-(1 / 2)((2 n$ $\left.+m+2)^{\lambda}+(2 n+m+2)^{\lambda}\right)+\sum_{m \equiv 0 \bmod }(2), 2 \leq m \leq 2 n-$ $2\left(\left((-m+2 n)^{3} / 24\right)+\left((-m+2 n)^{2} / 2\right)+5(-m+2 n) /\right.$ 6) $\left((2 n+m)^{\lambda}+(2 n+m)^{\lambda}\right)$

(iii) $h_{t}\left(R_{n}\right)=\left(3 n^{2}+4 n-1\right)(1 /(1+t))+\left(6 n^{2}+4 n-\right.$ 4) $(1 /(2+t))+\sum_{m \equiv 1 \bmod (2), 1<m \leq 2 n+1}\left(3 m n^{2}-2 m^{2} n+\right.$ $\left(7 m^{3} / 24\right)+6 m n-2 m^{2}+(41 m / 24)-$

1) $(1 /(m+t))+\sum_{m \equiv 0 \bmod (2), 2<m \leq 2 n}\left(3 m n^{2}-2 m^{n}+\right.$ $\left.\left(7 m^{3} / 24\right)+6 m n-2 m^{2}+(11 m / 6)-2\right)(1 /(m+t))+$ $\sum_{m \equiv 1 \bmod (2), 1 \leq m \leq 2 n-3}\left(\left((-m+2 n-2)^{3} / 24\right)+\quad((-m+\right.$ $\left.\left.2 n-2)^{2} / 2\right)+(23(-m+2 n-2) / 24)-(1 / 2)\right)(1 /$

$(2 n+m+2+t))+\sum_{m \equiv 0 \bmod (2), 2 \leq m \leq 2 n-2} \quad(((-m+$ $\left.\left.2 n)^{3} / 24\right)+\left((-m+2 n)^{2} / 2\right)+(5(-m+2 n) / 6)\right)$ $(1 /(2 n+m+t))$.

(iv) $\pi\left(R_{n}\right)=1^{\left(3 n^{2}+4 n-1\right)} \times 2^{\left(6 n^{2}+4 n-4\right)}$

$\prod_{m \equiv 1 \bmod (2), 1<m \leq 2 n+1}^{+} m\left(3 m n^{2}-2 m^{2} n+\left(7 m^{3} / 24\right)+\right.$ $\left.6 m n-2 m^{2}+(41 m / 24)-1\right) \times \prod_{m \equiv 0 \bmod (2), 2<m \leq 2 n}$ $m^{\left(3 m n^{2}-2 m^{n}+\left(7 m^{3} / 24\right)+6 m n-2 m^{2}+(11 m / 6)-2\right)} \times$

$\prod_{m \equiv 1 \bmod (2), 1 \leq m \leq 2 n-3}(2 n+m+2)^{\left(\left((-m+2 n-2)^{3} / 24\right)+\right.}$ $\left((-m+2 n-2)^{2} / 2\right)+(23(-m+2 n-2) / 24)-$ $(1 / 2)) \times \prod_{m \equiv 0 \bmod (2), 2 \leq m \leq 2 n-2}(2 n+m)$ $\left(\left((-m+2 n)^{3} / 24\right)+\left((-m+2 n)^{2} / 2\right)+5(-m+2 n) / 6\right)$

From the above results, we get following results immediately.

Corollary 4. For the benzenoid rhombus system $R_{n}$, we have

$$
W\left(R_{n}\right)=\frac{34 n^{5}}{15}+\frac{34 n^{4}}{3}+\frac{40 n^{3}}{3}+\frac{2 n^{2}}{3}-\frac{3 n}{5} \text {. }
$$

Corollary 5. For the benzenoid rhombus system $R_{n}$, we have

$W W\left(R_{n}\right)=42 n^{2}+32 n-26$

$$
\begin{aligned}
& +\sum_{m \equiv 1 \bmod (2), 1<m \leq 2 n+1} m(m+1)\left(3 m n^{2}-2 m^{2} n+\frac{7 m^{3}}{24}+6 m n-2 m^{2}+\frac{41 m}{24}-1\right) \\
& +\sum_{m \equiv 0 \bmod (2), 2<m \leq 2 n} m(m+1)\left(3 m n^{2}-2 m^{n}+\frac{7 m^{3}}{24}+6 m n-2 m^{2}+\frac{11 m}{6}-2\right) \\
& +\sum_{m \equiv \bmod (2), 1 \leq m \leq 2 n-3}\left(2 n+m+2+(2 n+m+2)^{2}\right) \times\left(\frac{(-m+2 n-2)^{3}}{24}+\frac{(-m+2 n-2)^{2}}{2}+\frac{23(-m+2 n-2)}{24}-\frac{1}{2}\right) \\
& +\sum_{m \equiv 0 \bmod (2), 2 \leq m \leq 2 n-2}\left(2 n+m+(2 n+m)^{2}\right)\left(\frac{(-m+2 n)^{3}}{24}+\frac{(-m+2 n)^{2}}{2}+\frac{5(-m+2 n)}{6}\right) .
\end{aligned}
$$


Corollary 6. The Harary index of benzenoid rhombus graph

$R_{n}$ is as follows:

$$
\begin{aligned}
h\left(R_{n}\right)= & 6 n^{2}+6 n-3+\sum_{m \equiv 1 \bmod (2), 1<m \leq 2 n+1} \frac{1}{m}\left(3 m n^{2}-2 m^{2} n+\frac{7 m^{3}}{24}+6 m n-2 m^{2}+\frac{41 m}{24}-1\right) \\
& +\sum_{m \equiv 0 \bmod (2), 2<m \leq 2 n} \frac{1}{m}\left(3 m n^{2}-2 m^{n}+\frac{7 m^{3}}{24}+6 m n-2 m^{2}+\frac{11 m}{6}-2\right) \\
& +\sum_{m \equiv 1 \bmod (2), 1 \leq m \leq 2 n-3} \frac{1}{2 n+m+2}\left(\frac{(-m+2 n-2)^{3}}{24}+\frac{(-m+2 n-2)^{2}}{2}+\frac{23(-m+2 n-2)}{24}-\frac{1}{2}\right) \\
& +\sum_{m \equiv 0 \bmod (2), 2 \leq m \leq 2 n-2} \frac{1}{2 n+m}\left(\frac{(-m+2 n)^{3}}{24}+\frac{(-m+2 n)^{2}}{2}+\frac{5(-m+2 n)}{6}\right) .
\end{aligned}
$$

\section{Conclusions}

Wiener demonstrated that the Wiener index is firmly connected to the boiling point of alkane. Later work on quantitative structure-activity connections demonstrated that it is additionally corresponded to different amounts including the parameters of its basic point the thickness, surface strain, and consistency of its fluid stage and the van der Waals surface territory of the molecules. Wiener index is a valuable topological index in the structureproperty relationship since it is the measurement of compactness of particle regarding its basic characteristics, for example, spreading and cyclicity. Utilizations of benzene follow a long history. In the nineteenth and midtwentieth centuries, benzene was utilized as an aftershave lotion due to its wonderful smell. Before the 1920 s, benzene was as often as possible utilized as a modern dissolvable, particularly to degrease metal. As its lethality wound up self-evident, benzene was displaced by different solvents, particularly toluene (methylbenzene), which has comparable physical properties yet is not as cancer-causing. In 1903, Ludwig Roselius promoted the utilization of benzene to decaffeinate espresso. This disclosure prompted the creation of Sanka. This procedure was later ended. Benzene was generally utilized as a noteworthy part in numerous shopper items, for example, Liquid Wrench, a few paint strippers, elastic concretes, spot removers, and different items. Produce of a portion of these benzene-containing details stopped in around 1950, albeit Liquid Wrench kept on containing critical measures of benzene until the late 1970s. In this present paper, we computed Hosoya polynomial, Wiener index, and hyper-Wiener index of zigzag and benzenoid rhombus systems. It is an interesting problem to find out distance-based topological indices for the families of graphs studied in [42-47].

\section{Data Availability}

All the data are included within this paper.

\section{Conflicts of Interest}

The authors of this paper declare that they have no conflicts of interest.

\section{Authors' Contributions}

All authors have equal contribution.

\section{Acknowledgments}

This research was funded by the Anhui Provincial Department of Education Natural Science Foundation (Project code: KJ2017A739).

\section{References}

[1] J.-B. Liu, C. Wang, S. Wang, and B. Wei, "Zagreb indices and multiplicative Zagreb indices of eulerian graphs," Bulletin of the Malaysian Mathematical Sciences Society, vol. 42, no. 1, pp. 67-78, 2019.

[2] J.-B. Liu, X.-F. Pan, F.-T. Hu, and F.-F. Hu, "Asymptotic Laplacian-energy-like invariant of lattices," Applied Mathematics and Computation, vol. 253, pp. 205-214, 2015.

[3] J.-B. Liu and X.-F. Pan, "Minimizing Kirchhoff index among graphs with a given vertex bipartiteness," Applied Mathematics and Computation, vol. 291, pp. 84-88, 2016.

[4] M. Munir, W. Nazeer, S. Rafique, and S. Kang, "M-polynomial and degree-based topological indices of polyhex nanotubes," Symmetry, vol. 8, no. 12, p. 149, 2016.

[5] S. Kang, Z. Iqbal, M. Ishaq, R. Sarfraz, A. Aslam, and W. Nazeer, "On eccentricity-based topological indices and polynomials of phosphorus-containing dendrimers," Symmetry, vol. 10, no. 7, p. 237, 2018.

[6] M. Ajmal, W. Nazeer, M. Munir, S. Kang, and Y. Kwun, "Some algebraic polynomials and topological indices of 
generalized prism and toroidal polyhex networks," Symmetry, vol. 9, no. 1, p. 5, 2016.

[7] A. Ali, W. Nazeer, M. Munir, and S. Min Kang, "M-polynomials and topological indices of zigzag and rhombic benzenoid systems," Open Chemistry, vol. 16, no. 1, pp. 73-78, 2018.

[8] W. Gao, B. Muzaffar, and W. Nazeer, "K-Banhatti and Khyper Banhatti indices of dominating David derived network," Open Journal of Mathematical Analysis, vol. 2017, no. 1, pp. 13-24, 2017.

[9] H. Wiener, "Structural determination of paraffin boiling points," Journal of the American Chemical Society, vol. 69, no. 1 , pp. 17-20, 1947.

[10] H. Hosoya, "On some counting polynomials in chemistry," Discrete Applied Mathematics, vol. 19, no. 1-3, pp. 239-257, 1988.

[11] I. Gutman, S. Klavzar, M. Petkovsek, and P. Zigert, "On Hosoya polynomials of benzenoid graphs," MATCH Communications in Mathematical and in Computer Chemistry, vol. 43, pp. 49-66, 2001.

[12] G. G. Cash, "Relationship between the Hosoya polynomial and the hyper-Wiener index," Applied Mathematics Letters, vol. 15, no. 7, pp. 893-895, 2002.

[13] W. Yan, B.-Y. Yang, and Y.-N. Yeh, "The behavior of Wiener indices and polynomials of graphs under five graph decorations," Applied Mathematics Letters, vol. 20, no. 3, pp. 290-295, 2007.

[14] M. R. Farahani, "On the Schultz polynomial and Hosoya polynomial of circumcoronene series of benzenoid," Journal of Applied Mathematics \& Informatics, vol. 31, no. 5-6, pp. 595-608, 2013.

[15] J.-B. Liu, J. Zhao, and Z. Zhu, "On the number of spanning trees and normalized Laplacian of linear octagonal-quadrilateral networks," International Journal of Quantum Chemistry, vol. 119, no. 17, Article ID e25971, 2019.

[16] J. Cao, J.-B. Liu, and S. Wang, "Resistance distances in corona and neighborhood corona networks based on Laplacian generalized inverse approach," Journal of Algebra and Its Applications, vol. 18, no. 3, Article ID 1950053,12 pages, 2019.

[17] J.-B. Liu, J. Zhao, J. Min, and J. Cao, "On the Hosoya index of graphs formed by a fractal graph," Fractals, vol. 27, no. 8, Article ID 1950135, 2019.

[18] Z. Zhu and J.-B. Liu, "The normalized Laplacian, degreeKirchhoff index and the spanning tree numbers of generalized phenylenes," Discrete Applied Mathematics, vol. 254, pp. 256-267, 2019.

[19] J.-B. Liu, S. Javed, M. Javaid, and K. Shabbir, "Computing first general Zagreb index of operations on graphs," IEEE Access, vol. 7, pp. 47494-47502, 2019.

[20] J. B. Liu, M. F. Nadeem, H. M. A. Siddiqui, and W. Nazir, "Computing metric dimension of certain families of Toeplitz graphs," IEEE Access, vol. 4, pp. 1-8, 2019.

[21] S. Nikolić, N. Trinajstić, and M. Randić, "Wiener index revisited," Chemical Physics Letters, vol. 333, no. 3-4, pp. 319-321, 2001.

[22] M. Ghorbani and S. Klavžar, "Modified Wiener index via canonical metric representation, and some fullerene patches," Ars Mathematica Contemporanea, vol. 11, no. 2, pp. 247-254, 2015.

[23] H. Shabani and A. R. Ashrafi, "The modified Wiener index of some graph operations," Ars Mathematica Contemporanea, vol. 11, no. 2, pp. 277-284, 2015.
[24] I. Lukovits and W. Linert, "A novel definition of the hyperWiener index for cycles," Journal of Chemical Information and Modeling, vol. 34, no. 4, pp. 899-902, 1994.

[25] M. Randić, "On generalization of Wiener index for cyclic structures," Acta Chimica Slovenica, vol. 49, no. 3, pp. 483496, 2002.

[26] G. Cash, S. Klavžar, and M. Petkovšek, "Three methods for calculation of the hyper-Wiener index of molecular graphs," Journal of Chemical Information and Computer Sciences, vol. 42, no. 3, pp. 571-576, 2002.

[27] W. F. Xi and W. Gao, " $\lambda$-modified extremal hyper-Wiener index of molecular graphs," Journal of Applied Computer Science \& Mathematics, vol. 18, no. 8, pp. 43-46, 2014.

[28] E. J. Farrell, "An introduction to matching polynomials," Journal of Combinatorial Theory, Series B, vol. 27, no. 1, pp. 75-86, 1979.

[29] M. R. Farahani, "On the Schultz and modified Schultz polynomials of some Harary graphs," International Journal of Applications of Discrete Mathematics, vol. 1, no. 1, pp. 1-8, 2013.

[30] S. Wagner, H. Wang, and X.-D. Zhang, "Distance-based graph invariants of trees and the Harary index," Filomat, vol. 27, no. 1, pp. 41-50, 2013.

[31] E. Estrada, "Three-dimensional generalized graph matrix, Harary descriptors, and a generalized interatomic LennardJones potential," The Journal of Physical Chemistry A, vol. 108, no. 25 , pp. $5468-5473,2004$

[32] I. Gutman, W. Linert, I. Lukovits, and Ž. Tomović, "The multiplicative version of the Wiener index," Journal of Chemical Information and Computer Sciences, vol. 40, no. 1, pp. 113-116, 2000.

[33] I. Gutman, W. Linert, I. Lukovits, and Ž. Tomović, "On the multiplicative Wiener index and its possible chemical applications," Chemical Monthly, vol. 131, no. 5, pp. 421-427, 2000.

[34] M. S. Anjum and M. U. Safdar, "K Banhatti and K hyperBanhatti indices of nanotubes," Engineering and Applied Science Letters, vol. 2, no. 1, pp. 19-37, 2019.

[35] Z. Shao, A. R. Virk, M. S. Javed, M. A. Rehman, and M. R. Farahani, "Degree based graph invariants for the molecular graph of Bismuth tri-iodide," Engineering and Applied Science, vol. 2, no. 1, pp. 1-11, 2019.

[36] A. U. R. Virk, M. N. Jhangeer, and M. A. Rehman, "Reverse zagreb and reverse hyper-Zagreb indices for silicon carbide $\mathrm{Si}_{2} \mathrm{C}_{3} I[r, s]$ and $\mathrm{Si}_{2} \mathrm{C}_{3} I I[r, s]$," Engineering and Applied Science Letters, vol. 1, no. 2, pp. 37-50, 2018.

[37] N. De, "Computing reformulated first Zagreb index of some chemical graphs as an application of generalized hierarchical product of graphs," Open Journal of Mathematical Sciences, vol. 2, no. 1, pp. 338-350, 2018.

[38] L. Yan, M. R. Farahani, and W. Gao, "Distance-based indices computation of symmetry molecular structures," Open Journal of Mathematical Sciences, vol. 2, no. 1, pp. 323-337, 2018.

[39] M. Imran, A. Asghar, and A. Q. Baig, "On graph invariants of oxide network," Engineering and Applied Science Letters, vol. 1, no. 1, pp. 23-28, 2018.

[40] W. Gao, A. Asghar, and W. Nazeer, "Computing degree-based topological indices of Jahangir graph," Engineering and Applied Science Letters, vol. 1, no. 1, pp. 16-22, 2018.

[41] R. Kanabu and S. Hosamani, "Some numerical invariants associated with V-phenylenic nanotube and nanotori," Engineering and Applied Science Letters, vol. 1, no. 1, pp. 1-9, 2018. 
[42] G. Liu, Z. Jia, and W. Gao, "Ontology similarity computing based on stochastic primal dual coordinate technique," Open Journal of Mathematical Sciences, vol. 2, no. 1, pp. 221-227, 2018.

[43] S. Noreen and A. Mahmood, "Zagreb polynomials and redefined Zagreb indices for the line graph of carbon nanocones," Open Journal of Mathematical Analysis, vol. 2, no. 1, pp. 66-73, 2018.

[44] Z. Tang, L. Liang, and W. Gao, "Wiener polarity index of quasi-tree molecular structures," Open Journal of Mathematical Sciences, vol. 2, no. 1, pp. 73-83, 2018.

[45] M. Riaz, W. Gao, and A. Qudair Baig, "M-polynomials and degree-based topological indices of some families of convex polytopes," Open Journal of Mathematical Sciences, vol. 2, no. 1, pp. 18-28, 2018.

[46] N. De, "Hyper Zagreb index of bridge and chain graphs," Open Journal of Mathematical Sciences, vol. 2, no. 1, pp. 1-17, 2018.

[47] H. Siddiqui and M. R. Farahani, "Forgotten polynomial and forgotten index of certain interconnection networks," Open Journal of Mathematical Analysis, vol. 1, no. 1, pp. 44-59, 2017. 